\title{
Intramarrow injection of $\beta$-catenin-activated, but not naïve mesenchymal stromal cells stimulates self-renewal of hematopoietic stem cells in bone marrow
}

\author{
Ji-Yeon Ahn ${ }^{1}$, Gyeongsin Park ${ }^{1}$, Jae-Seung Shim ${ }^{1}$, \\ Jong-Wook Lee ${ }^{2}$ and II-Hoan $\mathrm{Oh}^{1,3}$ \\ ${ }^{1}$ Catholic High-Performance Cell Therapy Center \\ Department of Cellular Medicine \\ ${ }^{2}$ Catholic Hematopoietic Stem Cell Transplantation Center \\ The Catholic University of Korea \\ Seoul 137-040, Korea \\ ${ }^{3}$ Corresponding author: Tel, 82-2-2258-8268; \\ Fax, 82-2-591-3994; E-mail, iho@ catholic.ac.kr \\ DOI 10.3858/emm.2010.42.2.014 \\ Accepted 1 December 2009 \\ Available Online 7 January 2010
}

Abbreviations: CFU-F, colony forming unit-fibroblast; CRU, competitive repopulating unit; HSC, hematopoietic stem cells; MSC, mesenchymal stromal cells

\begin{abstract}
Bone marrow mesenchymal stromal cells (MSCs) have been implicated in the microenvironmental support of hematopoietic stem cells (HSCs) and often co-transplanted with HSCs to facilitate recovery of ablated bone marrows. However, the precise effect of transplanted MSCs on HSC regeneration remains unclear because the kinetics of HSC self-renewal in vivo after co-transplantation has not been monitored. In this study, we examined the effects of intrafemoral injection of MSCs on HSC self-renewal in rigorous competitive repopulating unit (CRU) assays using congenic transplantation models in which stromal progenitors (CFU-F) were ablated by irradiation. Interestingly, naïve MSCs injected into femur contributed to the reconstitution of a stromal niche in the ablated bone marrows, but did not exert a stimulatory effect on the in-vivo self-renewal of co-transplanted HSCs regardless of the transplantation methods. In contrast, HSC self-renewal was four-fold higher in bone marrows intrafemorally injected with $\beta$-catenin-activated MSCs. These results reveal that naïve MSCs lack a stimulatory effect on HSC self-renewal in-vivo and that stroma must be activated during recoveries of bone marrows. Stromal targeting of wnt $/ \beta$-catenin signals may be a strategy to activate
\end{abstract}

such a stem cell niche for efficient regeneration of bone marrow HSCs.

Keywords: bone marrow; bone marrow transplantation; hematopoietic stem cells; stem cell niche; stromal cells

\section{Introduction}

Hematopoietic stem cells (HSC) are a rare cell population in the hematopoietic tissue that can sustain hematopoiesis throughout life and reconstitute bone marrows when transplanted into myeloablated recipients. The ability of transplanted HSCs to reconstitute the bone marrow for long-term period is dependent on their unique ability to execute selfrenewal during bone marrow regeneration (Lemischka et al., 1986; Eaves et al., 1997). Therefore, the execution of self-renewal in transplanted HSCs may determine the efficiency of bone marrow reconstitution and recovery of stem cell pools. The self-renewal of HSCs have been functionally defined by a quantitative increase in the number of transplantable stem cell referred to as competitive repopulating unit (CRU) (Szilvassy et al., 1990), and demonstration of CRU expansion through limiting dilution transplantation assays has been the most rigorous criteria of HSC self-renewal in-vivo (Pawliuk et al., 1996; Bhatia et al., 1997).

While several intrinsic regulators of HSC selfrenewal have been identified (Stein et al., 2004), recent studies have revealed a crucial role for the microenvironment in the self-renewal (Calvi et al., 2003; Zhang et al., 2003) and quiescence (Stier et al., 2005) of HSCs. The microenvironmental regulation of HSCs occurs in a specific architecture of the bone marrow stroma, called the stem cell niche. Recent studies have shown that there are two distinct types of compartments in the bone marrow stem cell niche, the endosteal osteoblastic compartment (Calvi et al., 2003; Zhang et al., 2003) and the peri-vascular compartment (Kiel and Morrison, 2006; Kiel et al., 2007). While the functional distinction between the two compartments remains unknown, it has recently been shown that mesenchymal stromal progenitors that can give rise to fibroblast colonies (colony forming unit-fibro- 
A

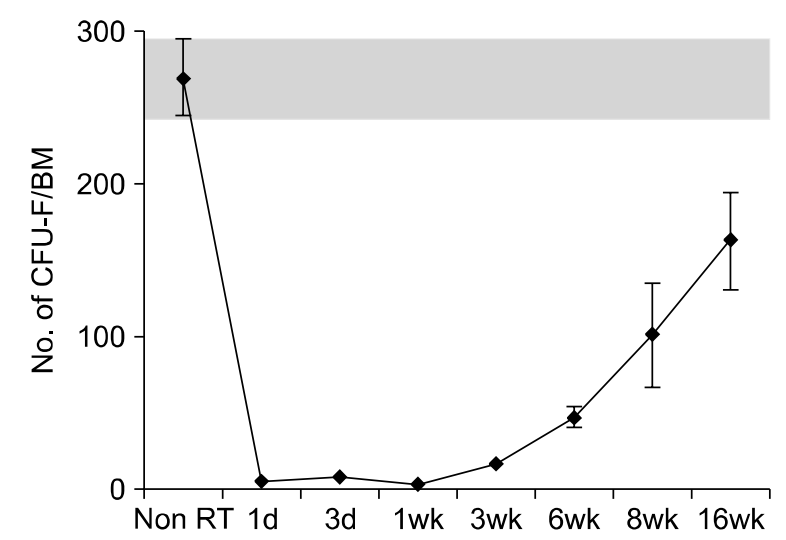

C

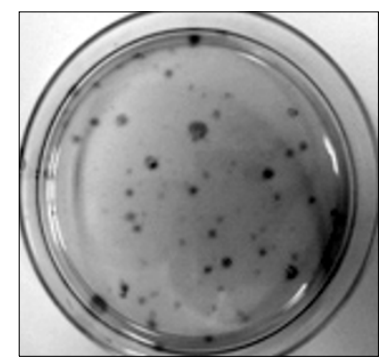

Non RT

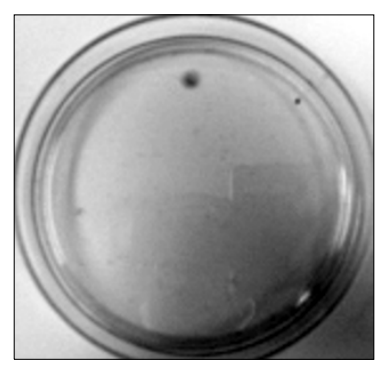

RT
B

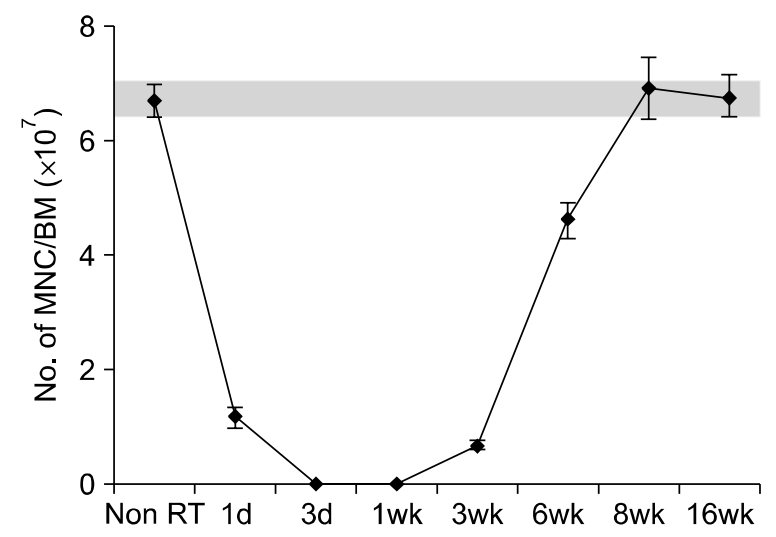

D

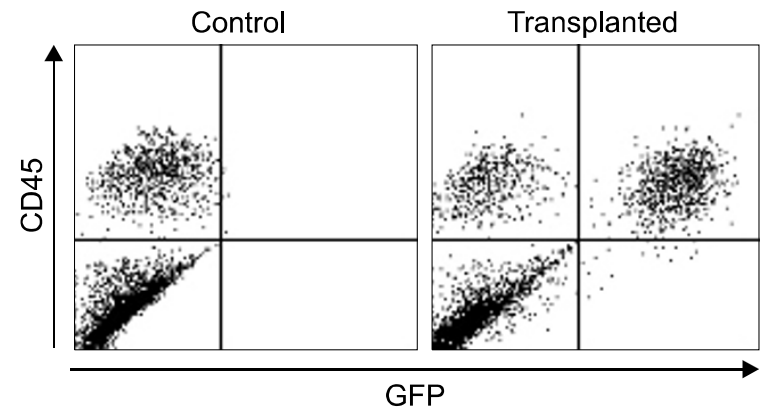

Figure 1. Recoveries of CFU-F in the irradiated bone marrows. Mice were total body irradiated (900 rad) and bone marrow cells $\left(1 \times 10^{5} / \mathrm{mice}\right)$ from transgenic mice expressing GFP were intravenously injected. Bone marrows (2 femurs and 2 tibias) of recipient mice were then harvested at various time points after irradiation and aliquots of the bone marrow cells were plated for 14 days to form CFU-F. The numbers of CFU-Fs formed in the plates were measured by crystal violet staining. Shown are the mean \pm SEM of CFU-F numbers (A) and total mononuclear cell (MNC) numbers (B) contained in two femurs and tibia of mice at each time point of recovery (3 independent experiments). (C) Representative image of crystal violet staining for CFU-F obtained after plating $1 \times 10^{7}$ bone marrow cells from non-radiated and irradiated ( $24 \mathrm{~h}$ before) mice. (D) Lack of stromal contribution from intravenously transplanted bone marrows cells. CFU-Fs generated in (A) were trypsinized and passage cultured to enrich non-hematopoietic (CD45-negative) stromal cells and examined for stromal cells of donor origin (CD45-neg, GFP + ). Shown are the representative flowcytometry profiles for subculture of CFU-Fs obtained from non-transplanted mice (control) and mice transplanted with GFP-transgenic mice bone marrow cells (transplanted).

blast; CFU-F) contribute to both the peri-vascular as well as the endosteal osteoblastic compartments of the stem cell niche (Sacchetti et al., 2007).

The mesenchymal stromal cells (MSCs) have been implicated in the supportive role for hematopoiesis, i.e. studies have shown that the co-culture of hematopoietic progenitors with MSCs results in a higher maintenance and expansion of HSCs (Kanai et al., 2000; Yamaguchi et al., 2001). In addition, co-transplantation of MSCs and HSCs facilitates hematopoietic engraftment of single or multiple-donor cells in xenogenic transplantations (Noort et al., 2002; Kim et al., 2004), and such approaches have begun to be applied in recent clinical transplantations (Ball et al., 2007; Le Blanc et al., 2007). Similarly, simultaneous injection of MSCs and HSCs directly into bone marrow has been shown to accelerate the recovery of hematopoietic cells in allogenic recipient mice (Zhang et al., 2004). However, because most co-transplantation studies until now were performed in the presence of an allogenic or xenogenic immune barrier, the possibility that the observed effects were due to the immune suppressive effects of MSCs (Le Blanc, 2006) has not been excluded. Moreover, the kinetics of self-renewal of HSCs after co-transplantation with MSCs has not been monitored in the previous studies and the effects of MSCs on HSC self- renewal remains unclear yet. Thus, despite the interests in MSCs during hematopoietic recovery, the precise biological effect of MSCs on HSCs in transplanted bone marrows remains largely unknown.

In the current study, HSCs were co-injected with MSCs into the bone marrow of congenic recipient 
A
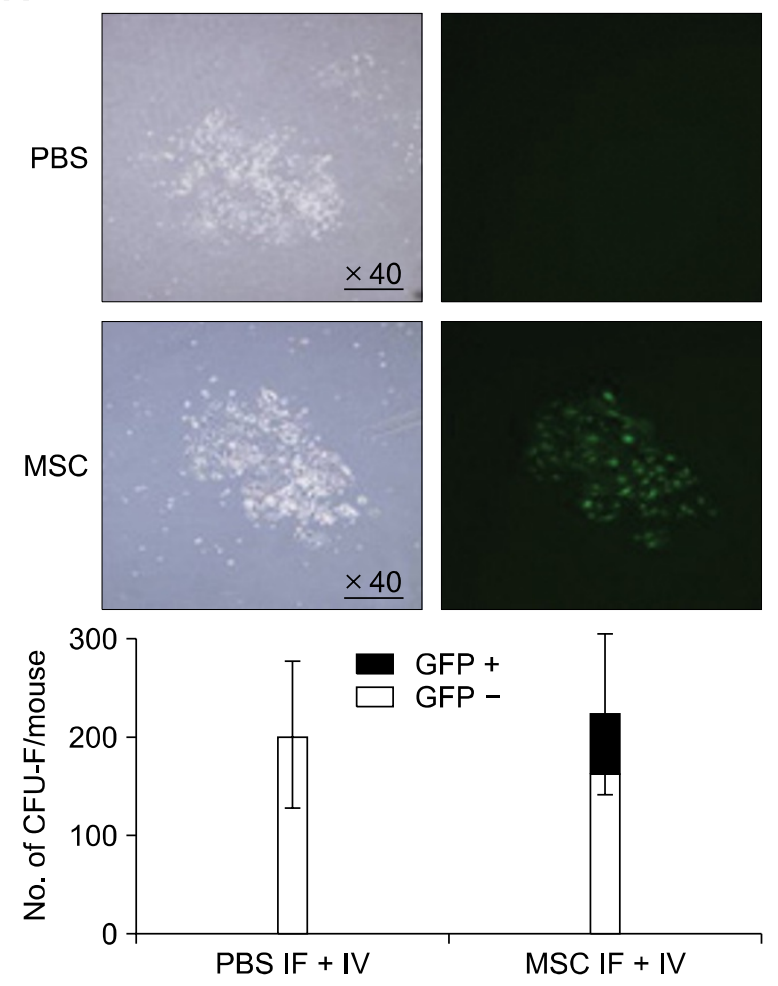

B
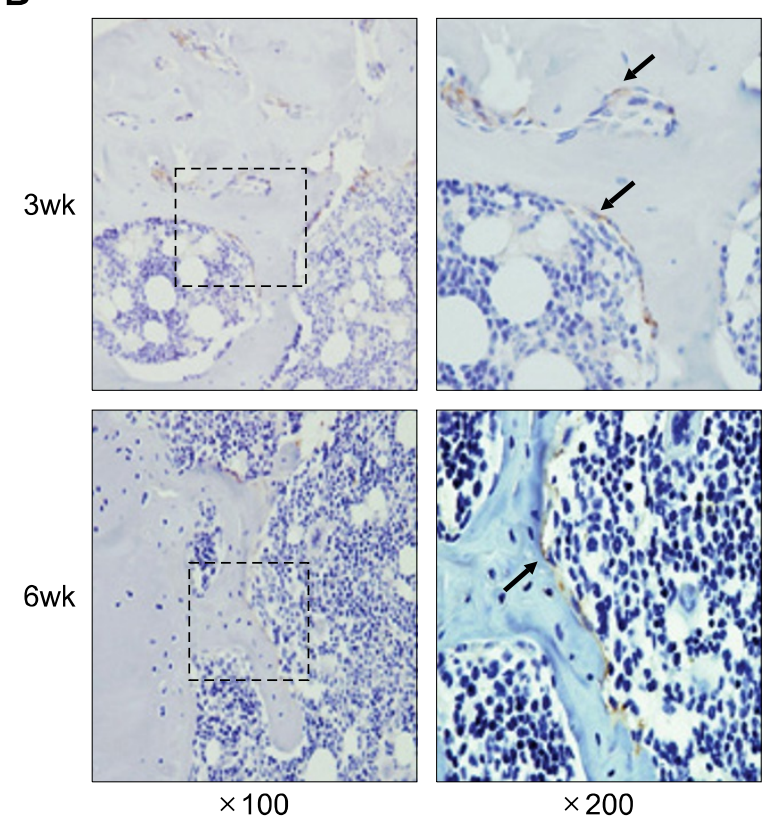

Figure 2. MSCs intrafemorally (IF) injected into bone marrows contribute to stromal regeneration in-vivo. (A) Contribution of injected MSCs to CFU-F pool of recipients. Mice were intrafemorally injected with PBS buffer or MSCs transduced with retroviral vector expressing GFP $(\mathrm{MPG})\left(1 \times 10^{5}\right.$ cells/ mice) and IV injected with helper cells $\left(1 \times 10^{5}\right.$ bone marrow cells/ mice). Recipient bone marrow cells were harvested 14 weeks after transplantation, plated and examined for GFP-positive CFU-F colonies by fluorescent microscope. Shown are representative photographs of GFP-positive colonies under light microscopy and fluorescence microscopy (upper) and the number of GFP-positive and GFP-negative CFU-F colonies (lower) ( $n=3$ ). (B) Bone marrows of mice injected with GFP-positive MSCs were immunostained with antibody against GFP at 3 and 6 weeks after transplantation. Shown are the representative images of trabecular bone marrows, at the indicated magnifications (dotted area), as visualized by DAB (brown). Arrows indicate positive staining for GFP.

mice that had been depleted of stromal progenitors (CFU-F) by total body irradiation and their selfrenewal was rigorously measured in a competitive repopulation assay. We show that naïve-state MSCs did not have stimulatory effects on HSCs. In contrast, $\beta$-catenin-activated MSCs promoted HSC self-renewal in the bone marrows thus providing insight into an "activated niche" for HSC regeneration.

\section{Results}

\section{CFU-F pools are rapidly destroyed after total body irradiation but can be reconstituted by ex-vivo cultured mesenchymal stromal cells}

We were first interested in the changes in the stromal microenvironment of bone marrow that can be induced by ablative total body irradiation. Quantitative changes in the number of CFU-F in bone marrow were used as a measure of stromal progenitor pools. Recipient mice (Pep 3b/Ly5.1) were total body irradiated (900 rad) and transplanted with bone marrow cells (BMCs) from congenic (BL6/Ly5.2) transgenic mice expressing the green fluorescent protein (GFP). Bone marrows were examined for CFU-Fs at various points during regeneration. As shown in Figure 1, the CFU-F content of the bone marrows decreased on the first day following irradiation, remained almost completely depleted ( $<10 \%$ of untreated control) for 3 weeks post-transplantation (Figure $1 \mathrm{~A}, \mathrm{C}$ ) and then began to recover. The regenerated CFU-F colonies were of recipient origin as reflected by the lack of GFP expression in non-hematopoietic (CD45negative) cells (Figure 1D). Of note, while the number of hematopoietic cells (total mononuclear cells) in irradiated bone marrows recovered to levels seen for control (un-irradiated) mice by 8 weeks after transplantation (Figure 1B), pools of CFU-F did not reach normal levels even after 16 weeks of regeneration (Figure 1A). These results show that following irradiation, stromal CFU-F pools are rapidly lost and remain depleted during 
A

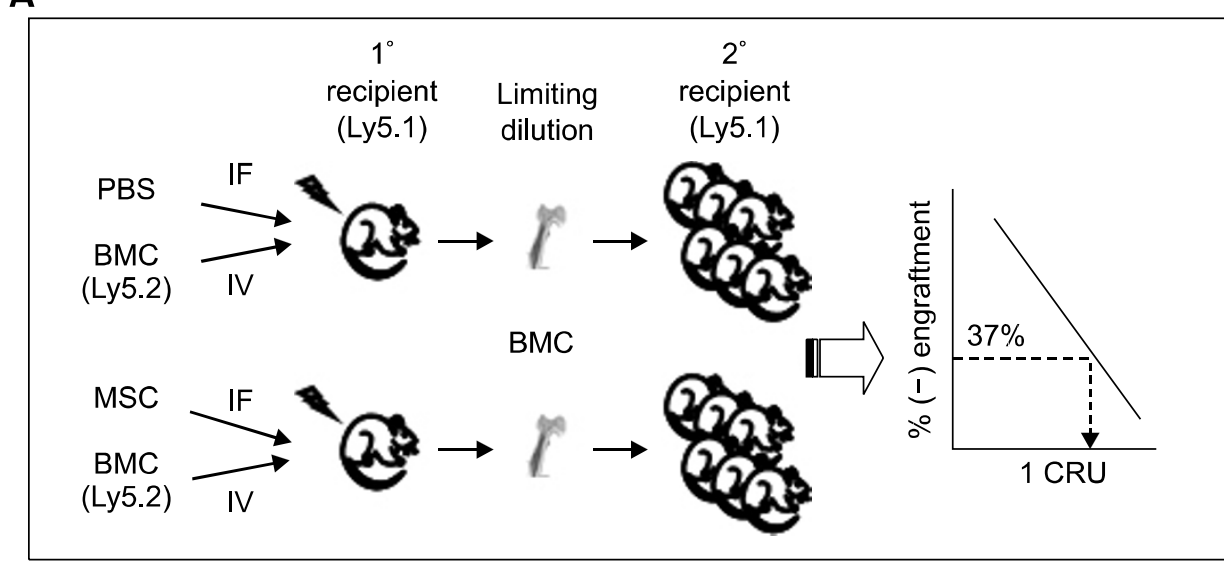

B

\begin{tabular}{crccc}
\hline Group & $\begin{array}{c}\text { Input } \\
\text { donor } \\
\text { cell dose }\end{array}$ & $\begin{array}{c}\text { Frequency of } \\
\text { reconstitution } \\
\left(2^{\circ} \text { recipient }\right)\end{array}$ & $\begin{array}{c}\text { CRU frequency } \\
(95 \% \text { C.I. })\end{array}$ & $\begin{array}{c}\text { CRU } \\
\text { /mouse } \\
(95 \% \text { C.I. })\end{array}$ \\
\hline PBS (IF) & 184,000 & $4 / 5$ & $1 / 180,000$ & 409 \\
+ & 36,800 & $0 / 5$ & $(1 / 474,000-68,000)$ & $(155-1,076)$ \\
BMC (IV) & 9,200 & $0 / 5$ & & \\
& & & $1 / 188,000$ & 540 \\
MSC (IF) & 254,000 & $4 / 5$ & $(1 / 466,000-1 / 76,000)$ & $(218-1,334)$ \\
+ & 50,800 & $1 / 5$ & & \\
BMC (IV) & 12,700 & $0 / 5$ & &
\end{tabular}

Figure 3. Effects of naïve MSCs on in vivo self-renewal of co-transplanted HSCs. Lethally irradiated mice (Ly5.1) were intravenously transplanted with congeneic (Ly5.2) donor bone marrow cells (BMCs) along with intrafemoral (IF) injection of MSCs or PBS buffer. Sixteen weeks after transplantation, the number of regenerated CRUs in the recipient mice was determined by limiting dilution transplantations of primary recipient mice marrow into secondary recipients. (A) Schematic illustration of the experimental design is shown. (B) The results of the $\mathrm{CRU}$ assays are shown. CRU frequencies and $95 \%$ confidence interval (C.I.) of the frequencies were obtained by applying Poisson statistics to the percent of negatively engrafted mice at different cell doses. The number of CRUs regenerated in mice was calculated based on the assumption that the cells in two femurs and tibias represent $25 \%$ of the total bone marrow as described (Boggs, 1984). the early phase of hematopoietic regeneration.

Having observed a decrease in the number of CFU-F pools following irradiation, we determined to examine the contribution of exogenous MSCs to reconstitution of bone marrow stroma. However, cultured MSCs transplanted through an i.v. route exhibit a limited homing into bone marrows (Rombouts and Ploemacher, 2003) and stromal cells mostly remained host origin after bone marrow transplantation (Garcia-Castro et al., 2007; Bartsch et al., 2009). Therefore, to circumvent the barrier, we chose to directly introduce cultured MSCs expressing GFP into bone marrows by intrafemoral injection, and bone marrow cells harvested from recovered mice were examined for CFU-F colonies from the injected MSCs (GFP +). As shown in Figure $2 \mathrm{~A}$, about $30 \%$ of total CFU-Fs regenerated in the recipient mice marrows were GFP+. Moreover, GFP positive cells were also observed in the trabecular endosteal region of the injected bone marrows when examined at 3 and 6 weeks after transplantation (Figure 2B). Thus, the injected MSCs contributed to the regeneration of
CFU-F as well as endosteal osteoblasts in the myeloablated bone marrows. Previous studies showed that the ability to regenerate these secondary CFU-Fs in the injected bone marrows comprise the characteristics of peri-vascular niche compartment (Sacchetti et al., 2007) and that endosteal osteoblast represents the osteoblastic niche (Calvi et al., 2003; Zhang et al., 2003). Together, these results showed that the cultured MSCs injected into femur contributed to the reconstitution of bone marrow stroma both in perivascular and endosteal niche as previously inferred (Muguruma et al., 2006; Sacchetti et al., 2007).

\section{Intrafemoral injection of naïve MSCs does not have stimulatory effects on the self-renewal of co-transplanted HSCs}

Based on the results that intrafemorally-injected MSCs contribute to stromal reconstitution, we next looked at whether MSCs introduced into bone marrow by intrafemoral injection can have an effect on the regenerative activity of HSCs. For this, we 
A

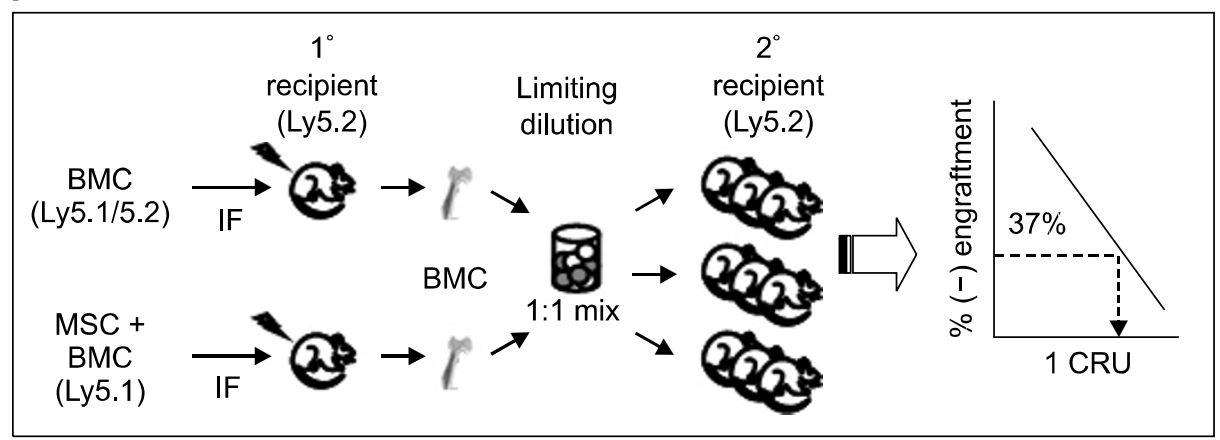

B

\begin{tabular}{ccccc}
\hline Group & $\begin{array}{c}\text { Input donor } \\
\text { cell dose }\end{array}$ & $\begin{array}{c}\text { Frequency of } \\
\text { reconstitution } \\
\left(2^{\circ} \text { recipient }\right)\end{array}$ & $\begin{array}{c}\text { CRU frequency } \\
(95 \% \text { C.I. })\end{array}$ & $\begin{array}{c}\text { CRU } \\
\text { /femur } \\
(95 \% \text { C.I. })\end{array}$ \\
\hline BMC & 780,000 & $5 / 5$ & & \\
(IF) & 420,000 & $8 / 9$ & $1 / 85,000$ & 156 \\
& 83,000 & $7 / 9$ & $(1 / 164,000-1 / 44,000)$ & $(81-301)$ \\
MSC & 45,000 & $4 / 5$ & & \\
+ & 810,000 & $5 / 5$ & $1 / 103,000$ & 121 \\
BMC & 410,000 & $8 / 9$ & $(1 / 197,000-1 / 54,000)$ & $(64-231)$ \\
(IF) & 83,000 & $7 / 9$ & & \\
\hline
\end{tabular}

Figure 4. Direct administration of HSCs and naive MSCs into femur as a mixture do not have stimulatory effects on the in-vivo self-renewal of HSCs. Effects of naive MSCs on HSCs were examined by directly transplanting HSCs and MSCs as a mixture into femur. The mixture of BMC (Ly5.1) plus MSCs or BMC alone ( $\mathrm{F} 1$ hybrid Ly5.1/5.2) were transplanted by intrafemoral (IF) injection into recipient mice. Eight weeks after transplantation, BMCs were harvested from the injected femurs of mice from each group $(n=$ 3 for each group), mixed together in a 1:1 ratio and subjected to limiting-dilution transplantations into secondary recipients. Engraftment of each donor origin cells in the same secondary recipient mice at 13 weeks after transplantation was discerned by surface marker (Ly5.1 or Ly5.1/5.2 hybrid). The experimental design $(A)$ and the results of the CRU analysis for the injected femurs (2 experiments) are shown (B). CRU frequencies with 95\% C.I. and CRU numbers were calculated as described in Methods.

examined the self-renewal of co-transplanted HSCs by measuring the number of competitive repopulating units (CRUs) regenerated in recipient mice. Recipients were intrafemorally injected with MSCs and concurrently i.v. injected with congenic BMCs. The number of regenerated CRUs of donor-origin in primary recipient mice was determined in a limiting dilution transplantation assay of primary recipient marrows into secondary recipient mice (Figure $3 \mathrm{~A}$ for experimental design).

Surprisingly, the number of donor-derived CRUs was not significantly different between the mice intrafemorally-injected with MSCs and those injected with PBS (409 CRUs for the PBS group and $540 \mathrm{CRUs}$ for the MSC group) (Figure 3B), indicating that MSCs injected intrafemorally did not exert any additional stimulatory effects on HSC self-renewal. However, a recent study has shown that distinct populations of hematopoietic progenitors can be engrafted through i.v. and intrafemoral routes of transplantation (Mazurier et al., 2003). In addition, i.v. and intrafemorally injected cells may not have sufficient opportunities for cellular interactions for enhancing effects to occur. Therefore, to rule out the possibility that the method of transplantation used in our experiment influenced the effect of MSCs on HSC self-renewal, we co-injected a mixture of MSCs and HSCs directly into the femur of recipient mice. As shown schematically in Figure 4A, recipient mice (Ly5.2) were transplanted by intrafemoral injection with either congenic BMCs alone (F1 Ly5.2/5.1 hybrid) or with both BMCs (Ly5.1) and MSCs. To compare HSC self- renewal under each condition, bone marrow cells reconstituted in each treatment group were harvested and mixed at a 1:1 ratio and analyzed for their relative quantity of CRUs in a competitive limiting- dilution transplantation assay into secondary recipient mice. As shown in Figure $4 \mathrm{~B}$, there was no difference in the number of CRUs in the bone marrow of mice injected with BMC alone and those injected with BMCs and MSCs (121 CRUs in the BMC + MSC femur vs. 156 CRUs in the BMC alone femur). Taken together, these results show that naïve-state MSCs, introduced into bone marrow by either i.v. or intrafemoral injection, do not exert a stimulatory effect on the self-renewal of co-transplanted HSCs.

\section{$\beta$-catenin-activated MSCs stimulate in-vivo self-renewal of co-transplanted HSCs}

The finding that naïve MSCs did not stimulate HSC self-renewal suggested that naïve MSCs intro- 
A

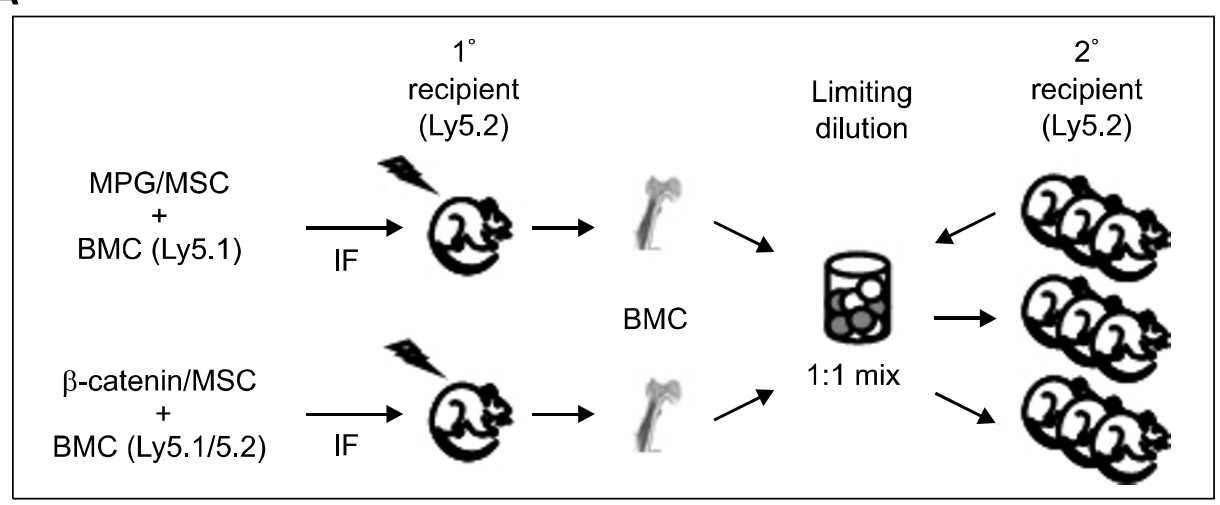

B

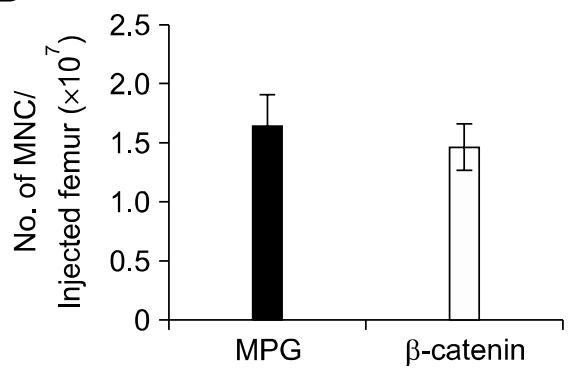

C

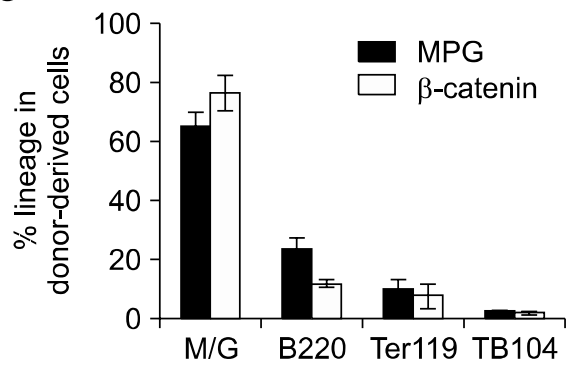

D

\begin{tabular}{|c|c|c|c|c|c|}
\hline Group & $\begin{array}{l}\text { Input donor } \\
\text { cell dose }\end{array}$ & $\begin{array}{l}\text { Frequency of } \\
\text { reconstitution } \\
\left.\text { ( } 2^{\circ} \text { recipient }\right)\end{array}$ & \multicolumn{2}{|c|}{$\begin{array}{l}\text { CRU frequency } \\
\quad(95 \% \text { C.I.) }\end{array}$} & $\begin{array}{c}\text { CRU } \\
\text { /femur } \\
(95 \% \text { C.I. })\end{array}$ \\
\hline $\begin{array}{c}\text { MPG } \\
/ \mathrm{MSC}+\mathrm{BMC}\end{array}$ & $\begin{array}{r}830,000 \\
420,000 \\
84,000 \\
42,000\end{array}$ & $\begin{array}{l}5 / 5 \\
7 / 9 \\
6 / 9 \\
2 / 6\end{array}$ & $\begin{array}{l}1 / 160,000 \\
(1 / 292,000 \\
-1 / 87,000)\end{array}$ & $1 \times$ & $\begin{array}{c}101 \\
(55-184)\end{array}$ \\
\hline $\begin{array}{c}\beta \text {-catenin } \\
/ \mathrm{MSC}+\mathrm{BMC}\end{array}$ & $\begin{array}{r}780,000 \\
360,000 \\
73,000 \\
34,000\end{array}$ & $\begin{array}{l}5 / 5 \\
9 / 9 \\
7 / 9 \\
5 / 6\end{array}$ & $\begin{array}{c}1 / 35,000 \\
(1 / 68,000 \\
-1 / 19,000)\end{array}$ & $4.5 \times$ & $\begin{array}{c}339 \\
(177-649)\end{array}$ \\
\hline
\end{tabular}

Figure 5 . Effects of $\beta$-catenin stabilized MSCs on the in vivo self-renewal of co-transplanted HSCs. Recipient mice were intrafemorally (IF) co-injected with a mixture of BMCs (Ly5.1) and MSCs transduced with MPG control vector (MPG/MSC), or with a mixture of BMCs (F1 hybrid Ly5.1/5.2) and MSCs transduced with stable form $\beta$-catenin ( $\beta$ - catenin/MSCs). Eight weeks after transplantation, the total number of MNCs in the injected bone marrows and lineage differentiation of the donor-derived cells were examined. BMCs harvested from the intrafemoral-injected femurs were pooled ( $n=6$ for each group) and pool of each treatment group were mixed in a 1:1 ratio and subjected to a competitive limiting-dilution transplantation into secondary recipients. Engraftment of each donor origin cells in the secondary recipient mice at 12 weeks after transplantation was discerned by surface marker (Ly5.1 or Ly5.1/5.2 hybrid). (A) Schematic illustration of the experimental design is shown. $(B, C)$ The total number of MNCs in the injected bone marrows of the primary recipient mice $(B)$ and the lineages of the engrafted cells $(C)$ are shown with error bars representing SEM $(n=3)$. (D) The results of the CRU analysis for the injected femurs are shown (2 experiments). CRU frequencies with $95 \%$ C.I. and total CRU numbers were calculated as described in Methods. duced by intrafemoral injection are not sufficient for stimulating the stromal microenvironment. We postulated that activated MSCs may be required to stimulate HSCs during early regeneration. Recently, we and another group showed that $\beta$-catenin is selectively activated in the stroma of stimulated bone marrows and that stromal $\beta$-catenin activity is required for the maintenance of HSCs during in vitro culture (Kim et al., 2009; Nemeth et al., 2009). The results of these studies raised the possibility that $\beta$-catenin activation in stroma creates an activated microenvironment for HSCs. Therefore, we wanted to test whether injection of $\beta$-catenin-activated MSCs, rather than naïve MSCs, into bone marrow could stimulate HSC self-renewal in the injected bone marrows.

To this end, we transduced MSCs with a stable form of $\beta$-catenin (Kim et al., 2009) or a control vector MPG and intrafemorally co-injected the transduced MSCs into recipient mice. The bone marrows regenerated under the two different conditions were mixed in a 1:1 ratio and analyzed by a competitive limiting-dilution transplantation assay in secondary recipients (schematically shown in Figure 5A). As shown in Figure 5D, the frequency of donor-derived CRUs regenerated with naïve MSCs was 1/16,0000 (1/292,000-1/87,000, 95\% $\mathrm{Cl})$ while the frequency observed with $\beta$-catenin- 
activated MSCs was 1/35,000 (1/68,000-1/19,000, $95 \% \mathrm{Cl}$ ), representing a 4.5 -fold higher frequency of CRUs regenerated with $\beta$-catenin-activated MSCs compared to naïve MSCs. Similarly, the total number of CRUs regenerated in each injected femur was higher in the group of mice injected with $\beta$-catenin-activated MSCs than in the group injected with naïve MSCs (100 CRUs vs. 339 CRUs for naïve MSCs and $\beta$-catenin/MSCs, respectively). These results show that $\beta$-catenin-activated MSCs, but not naïve-state MSCs, exert local stimulatory effects on HSC self-renewal in regenerating bone marrows. The increase of CRU frequency ( 3.8 folds) was similarly observed in the femurs of non-injected side in the mice injected with $\beta$ catenin/MSCs compared to control $(1 / 186,000$, vs $1 / 48,000$ for control and $\beta$-catenin/MSCs injected group, respectively) (data not shown), indicating that the enhancing effects are not confined to the MSC injected femur. However, the number of cells and the lineage of the regenerated bone marrow cells in the recipient mice injected with $\beta$-cateninactivated MSCs were not different from those injected with naïve MSCs (Figures $5 B$ and $5 C$ ). In addition, no signs of myeloproliferative disease were seen in the transplanted mice (data not shown). Taken together, these results show that injection of $\beta$-catenin-activated MSCs stimulates HSCs without causing changes to normal bone marrow cellularity, suggesting that stromal activation of Wnt/ $\beta$ - catenin signals may be a strategy for targeting the stem cell niche to create a stimulatory bone marrow microenvironment.

\section{Discussion}

Bone marrow stroma has been shown to be frequently destroyed during high-dose chemo/radiation therapy and loss of stromal progenitor cells has been associated with delayed recoveries of HSCs in bone marrow (Galotto et al., 1999; Ma et al., 2007). Thus it has been speculated that supplementation of bone marrow stromal cells into the myeloablated bone marrows could facilitate hematopoietic recoveries by transplanted HSCs. Supporting the speculation, positive effects were reported from in-vitro studies culturing stromal cells with HSCs, where higher level engraftments of HSCs (Kanai et al., 2000; Yamaguchi et al., 2001), or stimulatory cytokine effects of stromal cells on HSCs were observed (Verfaillie, 1993; FloresGuzman et al., 2009). However, it has not been clear whether similar stimulatory effects can be observed in-vivo by injection of MSCs into bone marrows. Moreover, most of previous studies on the co-transplantation of MSCs and HSCs was performed in the presence of immune barriers between the donor cells and the recipients (Noort et al., 2002; Ball et al., 2007; Le Blanc et al., 2007), thus making it difficult to distinguish the effects of the transplanted cells from the effects of immune suppression by MSCs. Thus, despite the increasing numbers of clinical studies adopting co-transplantation of culture-expanded MSCs and allogenic HSCs into patients (Ball et al., 2007; Le Blanc et al., 2007), the precise biological effects of MSCs on hematopoietic recoveries remain unclear, nor their possible effects on HSC self-renewal during the autologous HSC transplantations.

In the current study, we tracked the self-renewal of HSCs in vivo in bone marrows co-transplanted with MSCs using a congenic transplantation model. We used direct injection of MSCs into the femurs of recipient mice that had been almost completely depleted of CFU-F by irradiation. We found that intrafemoral injection of MSCs contributed to the regeneration of bone marrow stroma including peri-vascular and osteoblastic niche compartments. However, to our surprise, there were no additional stimulatory effects on HSC self-renewal in the bone marrows following the introduction of naïve MSCs by intrafemoral injection. Moreover, such lack of stimulatory effects on HSC self-renewal was consistently observed for HSCs transplanted through i.v. route or HSCs directly inoculated into femur as a mixture with MSC. These findings strongly suggested that naïve MSCs, in itself, do not exert a stimulatory effects on HSCs, and that MSCs must be activated to create a stimulatory microenvironment during the regeneration process.

In support of this hypothesis, we recently showed that $\beta$-catenin is activated in the stroma of bone marrows only under physiologically stimulated conditions (Kim et al., 2009). In the study, we showed that the notch ligands are induced in the $\beta$-catenin-stabilized MSCs and the down-stream notch signal is activated in the HSCs in contact with the activated MSCs. Similarly, another study demonstrated that stromal $\beta$-catenin activity is required for in vitro maintenance of long-term hematopoietic activity of HSCs (Nemeth et al., 2009). These findings led us to examine whether intrafemoral injection of $\beta$-catenin-stabilized MSCs instead of naîve MSCs can create an activated microenvironment to stimulate regenerative activity of HSCs. As seen in competitive CRU assays, direct injection of $\beta$-catenin-stabilized MSCs into the bone marrow increased HSC self-renewal four times above levels seen with injection of naïve MSCs, indicating that stromal activation of 
wnt/ $\beta$-catenin-activated MSCs was sufficient to promote in-vivo self-renewal of HSCs. Interestingly, CRU frequency was also increased in the noninjected side of femurs in the mice injected with $\beta$ catenin-activated MSCs despite that the MSCs did not migrate into other femurs. Thus it appears that the HSCs migrate beyond the locally injected femur to reach a systemic equilibrium in the enhancing effects of HSCs, as supported by previous finding that demonstrated rapid spreading of intrafemorally injected HSCs (Mazurier et al., 2003).

Of note, while the $\beta$-catenin-activated MSCs exerted a stimulatory enhancing effects on HSCs, the stimulatory effects did not override the normal limitations of bone marrow cellularity or lineage differentiation program of HSCs suggesting that the enhancing effects are under physiological regulation. Thus injection of $\beta$-catenin-activated MSCs instead of naïve MSCs during HSC transplantation can have therapeutic implication for physiological activation of HSC regenerative activity.

The microenvironment is increasingly becoming an attractive target for improving hematopoietic reconstitution (Adams and Scadden, 2008). In this light, strategies to activate $\mathrm{Wnt} / \beta$-catenin signals in the stroma and/or to reconstitute marrows with such activated stromal cells instead of naïve stromal cells may represent a model for the therapeutic targeting of the stem cell niche and efficient regeneration of HSCs.

\section{Methods}

\section{Animals and cell injection}

For the congenic murine transplantation model, 8 to 12 week-old C57BL/6J-Ly 5.2 (CD45.2) (BL6), C57BL/6JPep3b-Ly5.1 (CD45.1) (Pep3b) or their F1 hybrid (Ly5.1/ $5.2)$ mice were used as recipients or as donors. Mice were originally purchased from Charles River (Massachusetts, MA). Cells from these mice can be distinguished by their Ly5.1 or Ly5.2 surface phenotypes. Transgenic mice expressing GFP [C57BL6-Tg (CAG-EGFP)] were kindly provided by Dr. Okabe from the Osaka University, Japan. For injection of cells into mice, cells were injected either intrafemorally through the knee joint into the medullary cavity of right femur under anesthesia (Kushida et al., 2001; Mazurier et al., 2003) or injected intravenously. All animals were Pbred and maintained under sterile conditions in individually HEPA-filtered and ventilated cages in the animal facility of the Catholic University of Korea under the conditions of monitored temperature $\left(23.5^{\circ} \mathrm{C}\right)$, humidity $(54 \%)$, lightening $(12 \mathrm{~h})$ and positively pressured ventilation. Experiments were undertaken with approval from the Animal Experiment Board of the Catholic University of Korea.

\section{Cells}

Mesenchymal stromal cells (MSCs) were obtained by cul- turing murine bone marrow cells in DMEM containing $10 \%$ FBS (StemCell Technologies), as previously described (Kim et al., 2009). Established MSCs were subcultured until all cells became negative for the hematopoietic marker (CD45). In some experiments, MSCs were retrovirally transduced with a MPG vector expressing GFP under the phosphoglycerate kinase promoter (MSCV-PGKGFP; MPG) or a MPG vector harboring a stable form of the $\beta$-catenin gene (S37A), as previously described (Kim et al., 2009).

\section{In vitro assay for stromal progenitor cells (CFU-F) and bone marrow analysis}

To quantify the number of stromal progenitor cells (colony forming unit-fibroblasts; CFU-Fs), bone marrow cells were harvested by burr-hole punching, where femurs were punched by $21 \mathrm{G}$ syringe needle from the both ends (Kiel et al., 2007). Thus collected cells from the trabecular region and the central cavity were plated in a dish $\left(1 \times 10^{7}\right.$ cells/ $100 \mathrm{~mm}$ ), cultured for 2 weeks and visualized by crystal violet staining (Sigma-Adrich, St. Louis, MO). Detection of GFP-positive cells by immunostaining of bone marrows from the trabecular region was performed as previously described (Kim et al., 2009). Briefly, de-paraffinized slides were pre-treated with proteinase-K for antigen retrieval, blocked for endogeneous peroxidase, and incubated with antibody against GFP (Santa Cruz, Santa Cruz, CA) overnight at $4^{\circ} \mathrm{C}$, washed and incubated with a secondary antibody (HRP) for $30 \mathrm{~min}$ at room temperature and visualized with DAKO REAL ${ }^{\mathrm{TM}}$ EnVision $^{\mathrm{TM}}$ Detection System (DAKO, Glostrup, Denmark) and DAB, followed by hematoxylin counterstaining.

\section{In vivo repopulation and $\mathrm{CRU}$ assay}

Repopulation and differentiation of HSCs in congenic mice models was performed as previously described (Chung et al., 2006). For quantitative measurements of HSCs, CRU assays were performed as previously described (Szilvassy et al., 1990). Briefly, mice were lethally irradiated (900 cGy) by using ${ }^{137} \mathrm{Cs}$-irradiator $(3.42 \mathrm{~Gy} / \mathrm{min})$ and were transplanted with serial dilutions of cells and $1 \times 10^{5}$ helper cells. Recipient mice with $1 \%$ or more donor-lymphoid and myeloid engraftments were scored as positive. $1 \mathrm{CRU}$ was defined as the cell dose resulting in $37 \%$ of the mice tested being negative (Szilvassy et al., 1990). CRU frequencies and $95 \%$ confidence intervals (C.I.) were calculated by applying Poisson statistics to the proportion of negative mice from groups of recipients transplanted with different numbers of cells using L-Calc software (StemCell Technologies, Vancouver, Canada).

\section{Statistical analysis}

The significance of the difference was analyzed using the Student's $t$-test $(P<0.05)$ and the 95\% C.I. for the CRU frequency was calculated to represent \pm 2 SEM.

\section{Acknowledgements}

This research was supported by a grant of High-Perfor- 
mance Cell Therapy R\&D Project (0405-DB01-0104-0006) by the Ministry of Health and Welfare of the Republic of Korea, and in part by Korea Science and Engineering Foundation (KOSEF) (grant code: 2008-05981) (MEST).

\section{References}

Adams GB, Scadden DT. A niche opportunity for stem cell therapeutics. Gene Ther 2008;15:96-9

Ball LM, Bernardo ME, Roelofs H, Lankester A, Cometa A, Egeler RM, Locatelli F, Fibbe WE. Cotransplantation of ex vivo expanded mesenchymal stem cells accelerates lymphocyte recovery and may reduce the risk of graft failure in haploidentical hematopoietic stem-cell transplantation. Blood 2007; 110:2764-7

Bartsch K, Al-Ali H, Reinhardt A, Franke C, Hudecek M, Kamprad M, Tschiedel S, Cross M, Niederwieser D, Gentilini C. Mesenchymal stem cells remain host- derived independent of the source of the stem-cell graft and conditioning regimen used. Transplantation 2009;87:21721

Bhatia M, Bonnet D, Kapp U, Wang JC, Murdoch B, Dick, JE. Quantitative analysis reveals expansion of human hematopoietic repopulating cells after short-term ex vivo culture. J Exp Med 1997;186:619-24

Boggs DR. The total marrow mass of the mouse: a simplified method of measurement. Am J Hematol 1984;16:277- 86

Calvi LM, Adams GB, Weibrecht KW, Weber JM, Olson DP, Knight MC, Martin RP, Schipani E, Divieti P, Bringhurst FR, Milner LA, Kronenberg HM, Scadden DT. Osteoblastic cells regulate the haematopoietic stem cell niche. Nature 2003; 425:841-6

Chung YJ, Park BB, Kang YJ, Kim TM, Eaves CJ, Oh IH. Unique effects of Stat 3 on the early phase of hematopoietic stem cell regeneration. Blood 2006;108:1208-15

Eaves C, Miller C, Cashman J, Conneally E, Petzer A, Zandstra P, Eaves A. Hematopoietic stem cells: inferences from in vivo assays. Stem Cells 15 Suppl 1997;1:1-5

Flores-Guzman P, Flores-Figueroa E, Montesinos JJ, Martinez-Jaramillo G, Fernandez-Sanchez V, ValenciaPlata I, Alarcon-Santos G, Mayani H. Individual and combined effects of mesenchymal stromal cells and recombinant stimulatory cytokines on the in vitro growth of primitive hematopoietic cells from human umbilical cord blood. Cytotherapy, 2009;1-11

Galotto M, Berisso G, Delfino L, Podesta M, Ottaggio L, Dallorso S, Dufour C, Ferrara GB, Abbondandolo A, Dini G, Bacigalupo A, Cancedda R, Quarto R. Stromal damage as consequence of high-dose chemo/radiotherapy in bone marrow transplant recipients. Exp Hematol 1999;27:1460-6

Garcia-Castro J, Balas A, Ramirez M, Perez-Martinez A, Madero L, Gonzalez-Vicent M, Diaz MA. Mesenchymal stem cells are of recipient origin in pediatric transplantations using umbilical cord blood, peripheral blood, or bone marrow. J Pediatr Hematol Oncol 2007;29:388-92

Kanai M, Hirayama F, Yamaguchi M, Ohkawara J, Sato N,
Fukazawa K, Yamashita K, Kuwabara M, Ikeda H, Ikebuchi K. Stromal cell-dependent ex vivo expansion of human cord blood progenitors and augmentation of transplantable stem cell activity. Bone Marrow Transplant 2000;26:837-44

Kiel MJ, Morrison SJ. Maintaining hematopoietic stem cells in the vascular niche. Immunity 2006;25:862-4

Kiel MJ, Radice GL, Morrison SJ. Lack of evidence that hematopoietic stem cells depend on $\mathrm{N}$-cadherin- mediated adhesion to osteoblasts for their maintenance. Cell Stem Cell 2007;1:204-17

Kim DW, Chung YJ, Kim TG, Kim YL, Oh IH. Cotransplantation of third-party mesenchymal stromal cells can alleviate single-donor predominance and increase engraftment from double cord transplantation. Blood 2004;103: 1941-8

Kim JA, Kang YJ, Park G, Kim M, Park YO, Kim H, Leem SH, Chu IS, Lee JS, Jho EH, Oh IH. Identification of a stroma-mediated Wnt/beta-catenin signal promoting selfrenewal of hematopoietic stem cells in the stem cell niche. Stem Cells 2009;27:1318-29

Kushida T, Inaba M, Hisha H, Ichioka N, Esumi T, Ogawa R, lida $\mathrm{H}$, Ikehara $\mathrm{S}$. Intra-bone marrow injection of allogeneic bone marrow cells: a powerful new strategy for treatment of intractable autoimmune diseases in MRL/lpr mice. Blood 2001;97:3292-9

Le Blanc K. Mesenchymal stromal cells: Tissue repair and immune modulation. Cytotherapy 2006;8:559-61

Le Blanc K, Samuelsson H, Gustafsson B, Remberger M, Sundberg B, Arvidson J, Ljungman P, Lonnies H, Nava S, Ringden $O$. Transplantation of mesenchymal stem cells to enhance engraftment of hematopoietic stem cells. Leukemia 2007;21:1733-8

Lemischka IR, Raulet DH, Mulligan RC. Developmental potential and dynamic behavior of hematopoietic stem cells. Cell 1986;45:917-27.

Ma J, Shi M, Li J, Chen B, Wang H, Li B, Hu J, Cao Y, Fang $B$, Zhao RC. Senescence-unrelated impediment of osteogenesis from Flk1+ bone marrow mesenchymal stem cells induced by total body irradiation and its contribution to long-term bone and hematopoietic injury. Haematologica 2007;92:889-96

Mazurier F, Doedens M, Gan OI, Dick JE. Rapid myeloerythroid repopulation after intrafemoral transplantation of NOD-SCID mice reveals a new class of human stem cells. Nat Med 2003;9:959-63

Muguruma Y, Yahata T, Miyatake H, Sato T, Uno T, Itoh J, Kato $\mathrm{S}$, Ito M, Hotta T, Ando K. Reconstitution of the functional human hematopoietic microenvironment derived from human mesenchymal stem cells in the murine bone marrow compartment. Blood 2006;107:1878-87

Nemeth MJ, Mak KK, Yang Y, Bodine DM. beta- Catenin expression in the bone marrow microenvironment is required for long-term maintenance of primitive hematopoietic cells. Stem Cells 2009;27:1109-19

Noort WA, Kruisselbrink AB, in't Anker PS, Kruger M, van Bezooijen RL, de Paus RA, Heemskerk MH, Löwik CW, 
Falkenburg JH, Willemze R, Fibbe WE. Mesenchymal stem cells promote engraftment of human umbilical cord blood-derived CD34 (+) cells in NOD/SCID mice. Exp Hematol 2002;30:870-8

Pawliuk R, Eaves C, Humphries RK. Evidence of both ontogeny and transplant dose-regulated expansion of hematopoietic stem cells in vivo. Blood 1996;88:2852-8

Rombouts WJ, Ploemacher RE. Primary murine MSC show highly efficient homing to the bone marrow but lose homing ability following culture. Leukemia 2003;17:160-70

Sacchetti B, Funari A, Michienzi S, Di Cesare S, Piersanti S, Saggio I, Tagliafico E, Ferrari S, Robey PG, Riminucci M, Bianco P. Self-renewing osteoprogenitors in bone marrow sinusoids can organize a hematopoietic microenvironment. Cell 2007;131:324-36

Stein MI, Zhu J, Emerson SG. Molecular pathways regulating the self-renewal of hematopoietic stem cells. Exp Hematol 2004;32:1129-36

Stier S, Ko Y, Forkert R, Lutz C, Neuhaus T, Grünewald E, Cheng T, Dombkowski D, Calvi LM, Rittling SR, Scadden DT. Osteopontin is a hematopoietic stem cell niche component that negatively regulates stem cell pool size. J Exp Med 2005;201:1781-91
Szilvassy SJ, Humphries RK, Lansdorp PM, Eaves AC, Eaves CJ. Quantitative assay for totipotent reconstituting hematopoietic stem cells by a competitive repopulation strategy. Proc Natl Acad Sci USA 1990;87:8736-40

Verfaillie CM. Soluble factor(s) produced by human bone marrow stroma increase cytokine-induced proliferation and maturation of primitive hematopoietic progenitors while preventing their terminal differentiation. Blood 1993;82: 2045-53

Yamaguchi M, Hirayama F, Kanai M, Sato N, Fukazawa K, Yamashita K, Sawada K, Koike T, Kuwabara M, Ikeda H, Ikebuchi K. Serum-free coculture system for ex vivo expansion of human cord blood primitive progenitors and SCID mouse-reconstituting cells using human bone marrow primary stromal cells. Exp Hematol 2001;29:174-82

Zhang J, Niu C, Ye L, Huang H, He X, Tong WG, Ross J, Haug J, Johnson T, Feng JQ, Harris S, Wiedemann LM, Mishina Y, Li L. Identification of the haematopoietic stem cell niche and control of the niche size. Nature 2003;425:836-41

Zhang Y, Adachi Y, Suzuki Y, Minamino K, Iwasaki M, Hisha $\mathrm{H}$, Song CY, Kusafuka K, Nakano K, Koike Y, Wang J, Koh E, Cui Y, Li C, Ikehara S. Simultaneous injection of bone marrow cells and stromal cells into bone marrow accelerates hematopoiesis in vivo. Stem Cells 2004; 22:1256-62 